\title{
An Analytical Method for Quantifying Transport and Reaction of Anti-Tumor Drugs in Human Tissues
}

\author{
Dwaipayan MUKHERJEE \\ and Saikat CHAKRABORTY \\ Department of Chemical Engineering, Indian Institute of Technology, \\ Kharagpur, Kharagpur-721302, India
}

Keywords: Electroactive Polymers, Diffusion, Electric Potential, Drug Delivery, Anti-Tumor Drugs

\begin{abstract}
This work presents mathematical modeling and design of a novel implantable biocompatible polymer membrane-based controllable drug release device for delivery and uptake of anionic anti-tumor drugs. The diffusion-mediated transport of the drug from the drug reservoir to the affected tumor tissue is regulated by applying an electric potential across the membrane by a set of batteries. The model comprising of Poisson Boltzmann, Nernst Planck and Diffusion Reaction equations has been written for three separate compartments, namely, the drug reservoir, the polymer membrane and the diseased tissue, with the governing equations for the compartments being linked to each other through the boundary conditions. Analytical solutions of the abovedescribed three compartment model are obtained to quantify the drug delivery rate along the radial coordinate and with time. The analytical solution has been used to quantify the various controlling parameters and analyze the dynamics and efficacy of the drug delivery process for three different chemotherapeutic drugs, namely, Doxorubicin, Chlorambucil and Mitomycin C.
\end{abstract}

\section{Introduction}

Cancer is the third biggest killer among humans today, after cardiovascular and infectious diseases. But due to the multiple factors that cause cancer, and the rapid proliferation of the malignant tumor into healthy tissues, complete cancer therapy has still remained elusive. Chemotherapy has been recognized as a successful means of arresting this proliferation but the delivery of chemotherapeutic drugs offers a challenge: the proliferating cancer tissue requires a sustained delivery of the chemotherapeutic drug at regular intervals, while the healthy tissues must be protected from the chemical action of the drug. To ensure sustained yet targeted delivery, new and novel drug delivery mechanisms need to replace conventional ones.

This has resulted in previous attempts at designing novel, implantable, bio-compatible polymer-coated delivery devices that can release the drug at a specified rate at a particular location (Leong and Langer, 1987; Shivanand and Sprockel, 1998). We design and mathematically model drug delivery through a novel device that allows electrically regulated release of an ionic drug through an electroactive polymer membrane into a tumoraffected tissue. Our delivery device makes use of the fact that the electrical conductivity of electroactive polymers

Received on June 27, 2008; accepted on May 17, 2009. Correspondence concerning this article should be addressed to S. Chakraborty (E-mail address: dr.s.chakraborty@gmail.com).

Presented at ISCRE 20 in Kyoto, September, 2008. increases with applied electric field and is further increased several times by doping (Skotheim et al., 1998). Moreover, reversal of electric potential can make the membrane work like a switch by causing a reversal in oxidation states of the polymer units in the membrane (Bolto and Weiss, 1963).

Our drug delivery device consists of a cylindrical capsule comprising of a drug reservoir containing the ionic drug solution and a set of batteries for creating the desired electric field. The entire capsule is surrounded by a layer of selectively doped electroactive polymer. The sectional schematic of the delivery system (Figure 1) shows the three separate compartments for each of which the modeling is done separately - the drug reservoir of diameter $10 \mathrm{~mm}$, the polymer membrane of thickness $1 \mathrm{~mm}$ and the diseased tissue of thickness $15 \mathrm{~mm}$. The device is implanted at the centre of the tumor and the tumor is assumed to grow symmetrically around it. The anionic drug diffuses out under the action of the electric field, permeates through the electroactive membrane as it reacts with the charged polymer groups and is released into the diseased tissue, where it reacts with the tumor cells, killing them.

\section{Modeling}

The transport of the anionic drug into the affected tissue occurs due to three factors: the concentration gradient, the electric field created by the batteries, and the reversible reaction of the anionic drug (D) with the polymer groups $(\mathrm{P})$ inside the doped-polymer membrane to 


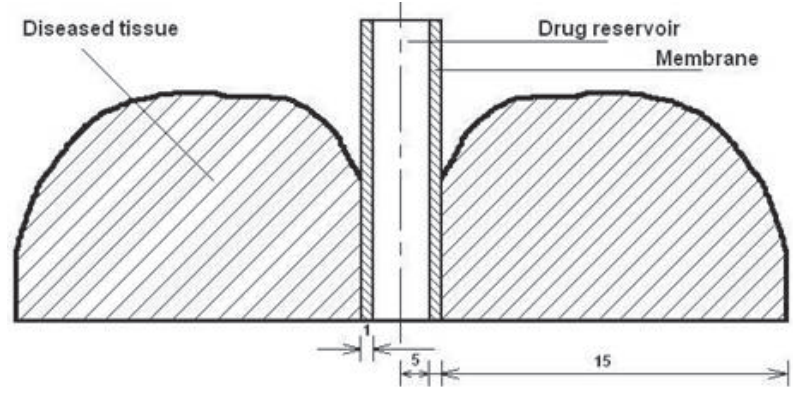

All dimensions in $\mathrm{mm}$

Fig. 1 Sectional view of the entire system

form a neutral complex (C), given by

$$
\mathrm{P}^{+}+\mathrm{D}^{-} \underset{k_{\mathrm{r}}}{\stackrel{k_{\mathrm{f}}}{\rightleftharpoons}} \mathrm{C}
$$

In our system, we split this reversible reaction into forward and backward reactions by applying alternate positive and negative voltage scans. During the positive scan, the presence of the dopant in the polymer causes it to be oxidized, generating positively charged polymer groups $\left(\mathrm{P}^{+}\right)$that attract the drug anions. The drug anions $\left(\mathrm{D}^{-}\right)$ enter the membrane and react with $\mathrm{P}^{+}$according to the forward reaction $\left(\mathrm{P}^{+}+\mathrm{D}^{-} \stackrel{k_{\mathrm{f}}}{\rightarrow} \mathrm{C}\right)$. No drug is able to diffuse out into the tissue during this period. During the negative scan, the polymer is reduced due to the reverse potential and hence can no longer hold on to the drug anions, resulting in the dissociation of the cationic polymer groups and drug anions, given by the reaction $\left(\mathrm{C} \stackrel{k_{\mathrm{r}}}{\rightarrow} \mathrm{P}^{+}+\mathrm{D}^{-}\right)$. Thus, the complex $\mathrm{C}$ dissociates to give back the polymer groups, and drug anions that diffuse out of the membrane into the surrounding tissue. However, some of the drug anions may also diffuse out back into the reservoir due to the reverse potential. Due to very high rate of release of the anions from the membrane, which is obviously higher than the rate of diffusion, a cluster of anions diffuse out to form in conjunction with the positively charged membrane surface, an "electric double layer" that behaves like a capacitor and creates an opposing electric field, preventing further reverse flux of the drug anions (Helfferich, 1962; Tanaka et al., 1980; Ricka and Tanaka, 1984).

\subsection{Potential distribution}

The potential field distribution in the reservoir and the membrane is dependent on the distribution of the ions in the medium and the strength of the fixed charges within the membrane, and is given by the Poisson Boltzmann equation as:

$$
\nabla^{2} \psi=-\frac{1}{\varepsilon}\left[\rho^{*}-2 q n_{0} \sinh (q \psi / k T)\right]
$$

where, $\psi$ is the potential distribution, $\rho^{*}$ is the fixed charge density $\rho$ of the surrounding membrane normalized over the entire space of the reservoir, i.e. $\rho^{*}=$ $\rho\left(v_{\mathrm{m}} / v_{\mathrm{s}}\right), v_{\mathrm{m}}$ and $v_{\mathrm{s}}$ are the volumes of the membrane and solution, respectively, $n_{0}$ is the number density of ions in the drug solution and $q$ is the basic unit of electric charge. In cylindrical coordinates, substituting $\hat{r}=r / R$, $\hat{z}=z / L, \phi=q \psi / k T$, where $R$ and $L$ are the radius and length of the reservoir respectively. Further noting that we $R \ll L$ obtain Eq. (1) in simplified form as:

$$
\begin{aligned}
& \frac{1}{R^{2}}\left(\frac{\partial^{2} \phi}{\partial \hat{r}^{2}}+\frac{1}{\hat{r}} \frac{\partial \phi}{\partial \hat{r}}\right) \\
& =-\frac{1}{\varepsilon}\left[\left(\frac{k T}{q}\right) \rho^{*}-2 k T n_{0} \sinh (\phi)\right]
\end{aligned}
$$

Since the battery generated potential in our device is small ( 0.1 to 1 volt), the weak field assumption is valid. Thus $\phi$ being small and $\sinh \phi \approx \phi$, we have

$$
\frac{\partial^{2} \phi}{\partial \hat{r}^{2}}+\frac{1}{\hat{r}} \frac{\partial \phi}{\partial \hat{r}}=A_{2} \phi-A_{1}
$$

where, $A_{1}=\left(\frac{R^{2} k T}{q \varepsilon}\right) \rho^{*}$ and $A_{1}=\left(\frac{R^{2} k T}{q \varepsilon}\right) \rho^{*}$

Taking $A_{2} \phi-A_{1}=y$ and $\hat{r} \sqrt{A_{2}}=\beta$, Eq. (3) could be rewritten as:

$$
\beta^{2} \frac{\partial^{2} y}{\partial \beta^{2}}+\beta \frac{\partial y}{\partial \beta}-\beta^{2} y=0
$$

with the boundary conditions being given by:

$$
\left.\frac{\partial y}{\partial \beta}\right|_{\beta=0}=0 \text {, and }\left.y\right|_{\beta=\sqrt{A_{2}}}=y_{1}
$$

where, $y_{1}$ denotes the value of $y$ at the membrane which is fixed by fixing the electrode potential. The solution of Eq. (4) is given by:

$$
y=c_{1} I_{0}(\beta)+c_{2} K_{0}(\beta)
$$

where $I_{\mathrm{o}}$ and $K_{\mathrm{o}}$ are modified Bessel functions of order zero. Applying the boundary conditions given by Eq. (5), we obtain the radial distribution of the electric potential $\psi$ as:

$$
\psi=\left(k T / A_{2} q\right)\left[y_{1} I_{0}(\alpha \theta) / I_{0}(\alpha)+A_{1}\right]
$$

where $\alpha=\sqrt{A_{2}}, \theta=r / R$.

\subsection{Transport in the reservoir}

The transport in the drug reservoir in the presence of the electric field is described by the Nernst Planck equation which is given by:

$$
\frac{\partial c_{\mathrm{D}}}{\partial t}+\nabla\left\{-D_{\mathrm{s}}\left[\left(\nabla c_{\mathrm{D}}\right)+\frac{z F}{k N_{\mathrm{A}} T} c_{\mathrm{D}}(\nabla \psi)\right]\right\}=0
$$

where $D_{\mathrm{s}}$ is the diffusivity of the drug in the reservoir solution, $z$ is the valence of the ion, $F$ is Faraday's constant and $N_{\mathrm{A}}$ is Avogadro's constant.

The assumptions made in solving Eq. (8) are (i) there is no depletion of drug within the reservoir, (ii) 
there are no convection effects in the system and (iii) the membrane is thin compared to the diameter of the reservoir. Due to assumption (iii), we use the potential gradient at the membrane i.e., at $r=R$, as constant throughout the thickness of the membrane and its value is given by:

$$
\left.\frac{d \psi}{d r}\right|_{r=R}=y_{1}(k T / q R \alpha) \frac{I_{1}(\alpha \theta)}{I_{0}(\alpha)}
$$

Substituting Eq. (9) in Eq. (8) and putting $z=-1$ (for anionic drug), we obtain

$$
\begin{aligned}
\frac{\partial c_{\mathrm{D}}}{\partial t}= & D_{\mathrm{s}}\left\{\frac{\partial^{2} c_{\mathrm{D}}}{\partial r^{2}}+\left[\frac{1}{r}-k_{1} \frac{I_{1}(\alpha \theta)}{I_{0}(\alpha)}\right] \frac{\partial c_{\mathrm{D}}}{\partial r}\right. \\
& \left.-k_{2} \frac{I_{1}{ }^{\prime}(\alpha \theta)}{I_{0}(\alpha)} c_{\mathrm{D}}\right\}
\end{aligned}
$$

where $k_{1}=F y_{1} / N_{A} R q \sqrt{A_{2}}$ and $k_{2}=k_{1} \sqrt{A_{2}} / R$.

1.2.1 Boundary layer solution Since the Bessel functions in Eq. (9) give very large values for most of the cylindrical reservoir, we solve for the drug concentration in a thin boundary layer adjacent to the reservoirmembrane interface, using Eq. (9). At the boundary we find that as:

$$
r \rightarrow R, \frac{I_{1}(\alpha \theta)}{I_{0}(\alpha)} \rightarrow 1 \text { and } \frac{I_{1}{ }^{\prime}(\alpha \theta)}{I_{0}(\alpha)} \rightarrow 1
$$

So Eq. (10) simplifies to

$$
\frac{\partial c}{\partial t}=D_{\mathrm{s}} \frac{\partial^{2} c}{\partial r^{2}}+D_{\mathrm{s}}\left(1 / r-k_{1}\right) \frac{\partial c}{\partial r}-D_{\mathrm{s}} k_{2} c
$$

(writing $c$ instead of $c_{\mathrm{D}}$ ), with boundary conditions given by

$$
\text { Atr }=R, c=c_{\mathrm{o}}, \text { Atr } \rightarrow-\infty, c \rightarrow c_{\mathrm{b}}
$$

Noting that $k_{1}, k_{2} \gg 1$ and $k_{1}, k_{2} \gg 1 /\left(R-r^{\prime}\right)$. Equation (11) simplifies to

$$
\frac{\partial c}{\partial t}=-D_{\mathrm{s}} k_{1} \frac{\partial c}{\partial r}-D_{\mathrm{s}} k_{2} c
$$

The solution for Eq. (12) and (13) is given by:

$$
c=c_{o}^{s}\left(t+(R-r) / D_{\mathrm{s}} k_{1}\right) \exp \left[\left(k_{2} / k_{1}\right)(R-r)\right]
$$

1.2.2 Electric double layer While the Nernst Planck Poisson (NPP) system of model equations given by Eqs. (1) and (8) is used to model the system during the positive scan, for the negative scan, the sign of the potential is reversed in the NPP equation. We also account for the electric double layer using the modified-Stern model (Lim et al., 2007) to quantify the effect of the electric double layer on the applied electric potential. It is found that for a solution of the strength we use here and for the applied voltage, the NPP system of equations predicts an electric field strength which is about 62 times than that given by the Nernst Planck Poisson Modified Stern (NPPMS) system of equations. Accordingly, the voltage strength given by the variable $y_{1}$ has to be reduced by this factor for the negative scan. As a result the values of $k_{1}$ and $k_{2}$ are reduced and at the same time their signs are reversed due to the reverse potential.

$$
\frac{\partial c}{\partial t}=D_{s} k_{1}^{\prime} \frac{\partial c}{\partial r}+D_{s} k_{2}^{\prime} c
$$

with boundary conditions given by

$$
\text { Atr }=R, c=c_{0}, \text { Atr } \rightarrow-\infty, c \rightarrow c_{\mathrm{b}}
$$

The solution for Eq. (16) is given by

$$
c=c_{0}^{s}\left(t-(R-r) / D_{\mathrm{s}} k_{1}^{\prime}\right) \exp \left[\left(k_{2}^{\prime} / k_{1}^{\prime}\right)(R-r)\right]
$$

\subsection{Transport in the polymeric membrane}

Since the membrane is very thin compared to the reservoir, we can assume the potential to follow a linear profile, with the same slope as that at the membranereservoir interface.

1.3.1 Positive scan The drug transport within the membrane is modeled with the same Nernst-Planck (NP) equation (with an extra reaction term being included due to the interaction between the drug and polymer group), which is given by:

$$
\begin{aligned}
\frac{\partial c_{\mathrm{D}}}{\partial t}= & \hat{D}_{\mathrm{m}}\left(\frac{\partial^{2} c_{\mathrm{D}}}{\partial r^{2}}+\frac{1}{r} \frac{\partial c_{\mathrm{D}}}{\partial r}\right) \\
& +D_{\mathrm{m}} \frac{z F s}{k N_{\mathrm{A}} T} \frac{\partial c_{\mathrm{D}}}{\partial r}-k_{\mathrm{f}} c_{\mathrm{p}} c_{\mathrm{D}}
\end{aligned}
$$

where $\hat{D}_{\mathrm{m}}=D_{\mathrm{m}}-R T k_{0}, D_{\mathrm{m}}$ being the diffusivity of the drug in the membrane, $k_{0}$ the osmotic coefficient of the ionic solution in the membrane, and $c_{\mathrm{p}}$ is the concentration of the polymer groups in the membrane.

Putting $z=-1$ and $c_{\mathrm{p}}=P_{0}$, the concentration of fixed charges in the polymer membrane,

$$
\begin{aligned}
\frac{\partial c_{\mathrm{D}}}{\partial t}= & \hat{D}_{\mathrm{m}}\left(\frac{\partial^{2} c_{\mathrm{D}}}{\partial r^{2}}+\frac{1}{r} \frac{\partial c_{\mathrm{D}}}{\partial r}\right) \\
& -D_{\mathrm{m}} \frac{F s}{k N_{\mathrm{A}} T} \frac{\partial c_{\mathrm{D}}}{\partial r}-k_{\mathrm{f}} P_{0} c_{\mathrm{D}}
\end{aligned}
$$

$\hat{D}_{\mathrm{m}} / r$ is of the order of $10^{-7}$ while $\left(D_{\mathrm{m}} F s\right) /\left(k N_{\mathrm{AT}}\right)$ is of the order of $10^{2}$, so neglecting the first two terms in the r.h.s. of Eq. (19), we have

$$
\frac{\partial c_{\mathrm{D}}}{\partial t}=-\frac{D_{\mathrm{m}} F s}{k N_{\mathrm{A}} T} \frac{\partial c_{\mathrm{D}}}{\partial r}-k_{\mathrm{f}} P_{0} c_{\mathrm{D}}
$$

with the boundary condition given by

$$
\text { At } r=R, c_{\mathrm{D}}=c_{0}
$$

The solution of Eq. (21) is given by

$$
c_{\mathrm{D}}=c_{0}\left(t-\frac{r^{\prime}}{\mu}\right) \exp \left(-\frac{k_{\mathrm{f}} P_{0}}{\mu} r^{\prime}\right)
$$

where $r^{\prime}=(r-R) / h$. 
1.3.2 Negative scan given by

$$
\begin{aligned}
& \frac{\partial c_{\mathrm{D}}}{\partial t}=\hat{D}_{\mathrm{m}}\left(\frac{\partial^{2} c_{\mathrm{D}}}{\partial r^{2}}+\frac{1}{r} \frac{\partial c_{\mathrm{D}}}{\partial r}\right)-D_{\mathrm{m}} \frac{z F s}{k N_{\mathrm{A}} T} \frac{\partial c_{\mathrm{D}}}{\partial r}+k_{\mathrm{r}} c_{\mathrm{c}} \\
& \frac{\partial c_{\mathrm{c}}}{\partial t}=D_{\mathrm{c}}\left(\frac{\partial^{2} c_{\mathrm{D}}}{\partial r^{2}}+\frac{1}{r} \frac{\partial c_{\mathrm{D}}}{\partial r}\right)-k_{\mathrm{r}} c_{\mathrm{c}}
\end{aligned}
$$

where $\hat{D}_{m}=D_{m}-R T k_{0}, c_{\mathrm{D}}$ is drug concentration and $c_{\mathrm{c}}$ is the concentration of the complex C. Neglecting the first two terms in Eq. (24) due to smallness as explained for the previous case, we have

$$
\frac{\mathrm{d} c_{\mathrm{c}}}{\mathrm{d} t}=-k_{\mathrm{r}} c_{\mathrm{c}}
$$

the solution of which is given by

$$
c_{\mathrm{c}}=c_{1} \exp \left(-k_{\mathrm{r}} t\right)
$$

where the initial concentration of the complex, $c_{1}(r)$ is obtained from the curve at the end of the positive scan by substituting for $t=t_{\mathrm{p}}$ (time of positive scan) in Eq. (22) as:

$$
c_{1}\left(r^{\prime}\right)=\left(t_{p}-\frac{r^{\prime}}{\mu}\right) \exp \left(-\frac{k_{f} P_{0}}{\mu} r^{\prime}\right)
$$

Substituting the result in Eq. (23), neglecting the first two terms on the r.h.s as in the last section and further putting $r^{\prime}=(r-R) / h$ and $\mu^{\prime}=\left(D_{\mathrm{m}} F s^{\prime}\right) /(R T h)$ the solution of Eq. (23) is obtained as:

$$
c_{D}=c_{0}\left(t_{p}+\frac{r^{\prime}}{\mu}\right)+f\left(r^{\prime}\right) \exp \left(-k_{r} t-\frac{k_{f} P_{0}}{\mu^{\prime}} r^{\prime}\right)
$$

where $f\left(r^{\prime}\right)=\left(1 / k_{3}\right)\left[k_{\mathrm{r}}\left(t_{\mathrm{p}}-r^{\prime} / \mu^{\prime}\right)-\left(k_{r} / k_{3}\right)\right]$ and $k_{3}=k_{\mathrm{r}}-k_{\mathrm{f}} P_{0}$.

\subsection{Transport and drug reaction in the tumor- affected tissue}

The transport and reaction of the drug is modeled by the diffusion-reaction equation. The dimensions of the tissue being much larger than that of the drug reservoir, its radius of curvature could be neglected and the governing equation could be expressed as:

$$
\frac{\partial c_{\mathrm{D}}}{\partial t}=D \frac{\partial^{2} c_{\mathrm{D}}}{\partial r^{2}}+R_{\mathrm{D}}
$$

where $R_{\mathrm{D}}$, the reaction rate of the drug is estimated by Michaelis-Menten kinetics as:

$$
R_{\mathrm{D}}=\frac{v_{\max } c_{\mathrm{D}}}{c_{\mathrm{D}}+K_{\mathrm{m}}}
$$

which for low values of concentration reduces to first order kinetics with $k=v_{\max } / K_{\mathrm{m}}$ being the rate constant.
Equation (29) then reduces to

$$
\frac{\partial c_{\mathrm{D}}}{\partial t}=D \frac{\partial^{2} c_{\mathrm{D}}}{\partial r^{2}}-k c_{\mathrm{D}}
$$

Equation (31) is solved separately for the positive and negative scans. The analytical solution is obtained with the help of the Finite Fourier method. We model the negative scan first followed by the positive scan and use the temporal continuity between the two scans.

1.4.1 Negative scan It is assumed that any drug present as a residue of the earlier negative scan is completely consumed in the consecutive positive scan. So the initial concentration $\mathrm{c}^{\circ}$ is taken as zero. The boundary condition at $x=0$ is obtained from the concentration profile in the polymeric membrane during the negative scan. Putting $r^{\prime}=1$ in Eq. (28), we have

$$
c_{\mathrm{D}}=c_{0}\left(t+\frac{1}{\mu}\right)+k_{4} \exp \left(-k_{\mathrm{r}} t-\frac{k_{\mathrm{f}} P_{0}}{\mu}\right)
$$

which reduces to

$$
c_{\mathrm{D}} \approx c_{0}\left(t+\frac{1}{\mu}\right)
$$

which is approximated by an exponential expression for ease of the analytical solution as

$$
c_{0}\left(t+\frac{1}{\mu}\right) \approx a-b\left(e^{-\alpha t}-1\right)
$$

where $a=c_{0}, b=400 c_{0}$ and $\alpha=0.0025$.

The concentration at $r=1$, is also taken as a function of time as:

$$
c(1, t)=c_{\mathrm{T}}\left[1+(\gamma-1) e^{-\beta t}\right]
$$

where $c_{\mathrm{T}}$ is the therapeutic concentration of the drug.

So the boundary condition may be written as:

$$
c(1, t)=a_{1}+b_{1} e^{-\beta_{1} t}
$$

where $a_{1}=c_{\mathrm{T}}$ and $b_{1}=c_{\mathrm{T}}(\gamma-1)$.

So Eq. (31) is to be solved with the following initial and boundary conditions

$$
\begin{aligned}
& c(r, 0)=0, c(0, t)=a-b\left(e^{-\alpha t}-1\right), \\
& c(1, t)=a_{1}+b_{1} e^{-\beta_{1} t}
\end{aligned}
$$

The solution obtained by the Finite Fourier Transform Method, after putting the boundary conditions is

$$
\begin{aligned}
c_{\mathrm{D}}(r, t)= & \left(a+b-b e^{-\alpha t}\right)(1-r)+\left(a_{1}+b_{1} e^{-\beta t}\right) r \\
& +\sum_{n=1}^{\infty}\left\{\frac{2 D n \pi \sin (n \pi r)}{P_{\mathrm{n}}}\right. \\
& \left.\cdot\left[(-1)^{n}\left(a_{1}+b_{1}\right)-a\right] e^{-} P_{n} \mathrm{t}\right\}
\end{aligned}
$$

where, $P_{n}=D n^{2} \pi^{2}+k, a_{1}=a+b$ and $\alpha=\beta=k$. 
1.4.2 Positive scan The modeling for the positive scan is carried out in much the same way as in the negative scan, but we use a Neumann boundary condition at the membrane-tissue interface and a Dirichlet condition at the other boundary. This is because, during the positive scan there is no release of drug ions from the membrane and so transfer of drug ions from the membrane to the tissue is zero. The spatial concentration profile at the end of the negative scan is used as the initial condition for the positive scan. Putting $t=t_{\mathrm{n}}$ (the time for negative scan) in Eq. (38), we have

$$
\begin{aligned}
& c\left(r, t_{\mathrm{n}}\right) \\
& =\left[a+b\left(1-e_{\mathrm{n}}^{-\alpha t}\right)-\left(a_{1}+b_{1} e^{-\beta_{1} t_{\mathrm{n}}}\right)\right](1 / 2-r) \\
& +\left[a+b\left(1-e^{-\alpha t_{\mathrm{n}}}\right)+\left(a_{1}+b_{1} e^{-\beta_{1} t_{\mathrm{n}}}\right)\right](1 / 2) \\
& -\sum_{n=1}^{\infty}\left[\frac{2 D n \pi \sin (n \pi r)}{P_{n}}\left\{a-(-1)^{n}\left(a_{1}+b_{1}\right)\right\} e^{-} P_{n} t_{\mathrm{n}}\right]
\end{aligned}
$$

which reduces to

$$
c\left(r, t_{\mathrm{n}}\right) \approx g+h r
$$

where $g=a+b\left(1-e_{n}^{-\alpha t}\right)$ and

$$
h=\left(a_{1}+b_{1} e^{-\beta_{1} t_{\mathrm{n}}}\right)-a-b\left(1-e^{-\alpha t_{\mathrm{n}}}\right)
$$

So Eq. (31) is to be solved with the following initial and boundary conditions

$$
\begin{aligned}
& c(r, 0)=f(r)=g+h r \\
& c^{\prime}(0, t)=0, c(1, t)=a_{1}+b_{1} e^{-\beta t}
\end{aligned}
$$

The solution is obtained in a similar way, and after substituting the relevant boundary conditions, we obtain

$$
\begin{aligned}
& c_{\mathrm{D}}(r, t) \\
& =b_{1} e^{-\beta t}-2 \sum_{n=1}^{\infty}\left[\frac{h \cos (n-1 / 2) \pi r}{(n-1 / 2)^{2} \pi^{2}} e^{-} M_{n} t\right] \\
& -2 \sum_{n=1}^{\infty}\left[(-1)^{n} \frac{(g+h) \cos (n-1 / 2) \pi r}{(n-1 / 2) \pi} e^{-} M_{n} t\right] \\
& +2 \sum_{n=1}^{\infty}\left[(-1)^{n} \frac{\cos (n-1 / 2) \pi r}{(n-1 / 2) \pi} b_{1} e^{-} M_{n} t\right]
\end{aligned}
$$

where, $M_{n}=D(n-1 / 2)^{2} \pi^{2}+k$ and $\beta=k$.
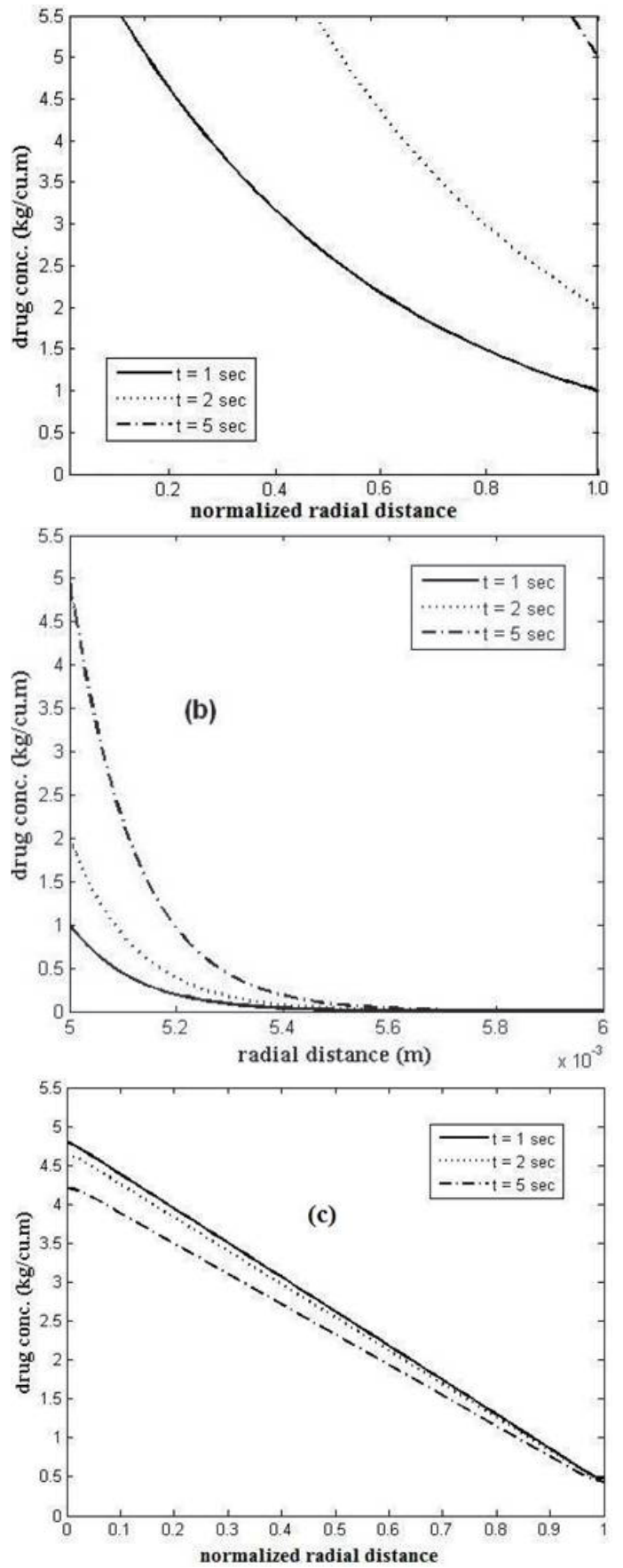

Fig. 2 Drug concentration plots during the positive scan in the (a) reservoir, (b) membrane and (c) tissue (Radial distance is from center of reservoir)

\section{Results and Discussion}

The results are shown for the three compartment model having the drug reservoir, the membrane and the tumor-affected tissue. Diffusivity of the drug in the three zones - the reservoir, the membrane and the tissue are $1.6 \times 10^{-6}, 1 \times 10^{-9}$ and $1.2 \times 10^{-8} \mathrm{~m}^{2} / \mathrm{s}$, respectively. The voltage scan times for both positive and negative scans are taken as $5 \mathrm{~s}$. Drug concentration in the reser- 
Table 1 Summary of kinetic constants

\begin{tabular}{ll}
\hline Kinetic constants & Value \\
\hline$k_{\mathrm{f}}$ & $20 \mathrm{~m}^{3} /(\mathrm{mol} \mathrm{s})$ \\
$k_{\mathrm{r}}$ & $3.6 \times 10^{3} \mathrm{~s}^{-1}$ \\
$K_{\text {doxo }}$ & $1.28 \times 10^{-5} \mathrm{~s}^{-1}$ \\
$K_{\text {cloram }}$ & $1.28 \times 10^{-4} \mathrm{~s}^{-1}$ \\
$K_{\text {mito }}$ & $4.12 \times 10^{-4} \mathrm{~s}^{-1}$ \\
\hline
\end{tabular}

voir solution is $0.1 \mathrm{M}$ and the dopant concentration in the membrane is $10 \mathrm{M}$. The membrane chosen is polypyrrole (Diaz et al., 1979; Frank et al., 2005). The drug and biological data conform to the diffusion of the anti-tumor drug doxorubicin in rat-liver. The various kinetic constants are summarized in Table $\mathbf{1}$.

\subsection{Drug concentration}

Figures $\mathbf{2}$ and $\mathbf{3}$ show the plots of drug concentration in the three compartments at different times. The fast disappearance of drug in the membrane in the positive scan is due to the fast ionic reaction that converts the drug into the neutral complex. The reverse reaction is also fast and hence a constant profile is obtained in the same region during the negative scan, as the fast evolving anions are unable to diffuse out.

\subsection{Parametric study}

A parametric study has been performed for the positive scan in the membrane (since the drug is taken into the membrane only during the positive scan). The final release of the drug into the tissue would depend on the amount of drug immobilized in the membrane. The time has been kept constant at 2 seconds for all the parametric studies.

Figure 4(a) shows expected results. The drug concentration increases with increase in diffusivity of the polymeric membrane. Figure 4(b) shows an increase in drug concentration with increasing thickness which occurs since the number of polymer groups within the membrane increases allowing the membrane to accept more drug anions.

Figure 4(c) shows that the drug delivery increases with voltage, allowing us to use voltage as a regulating factor in the delivery, though within physiological limit ( $=1$ volt $)$.

The behavior of two other chemotherapeutic drugs, Chlorambucil (Zhang et al., 2004) and Mitomycin C (Gustafson and Pritsos, 1993) has also been considered. They both have first order rate constants higher than that of doxorubicin as a result of which they get depleted at a much higher rate in the tumor, as seen in Figure 4(d), with Mitomycin $\mathrm{C}$ exhibiting the lowest concentration level in the tissue.

\section{Conclusions}

Our parametric study that quantifies the drug concentration levels under different conditions provides an
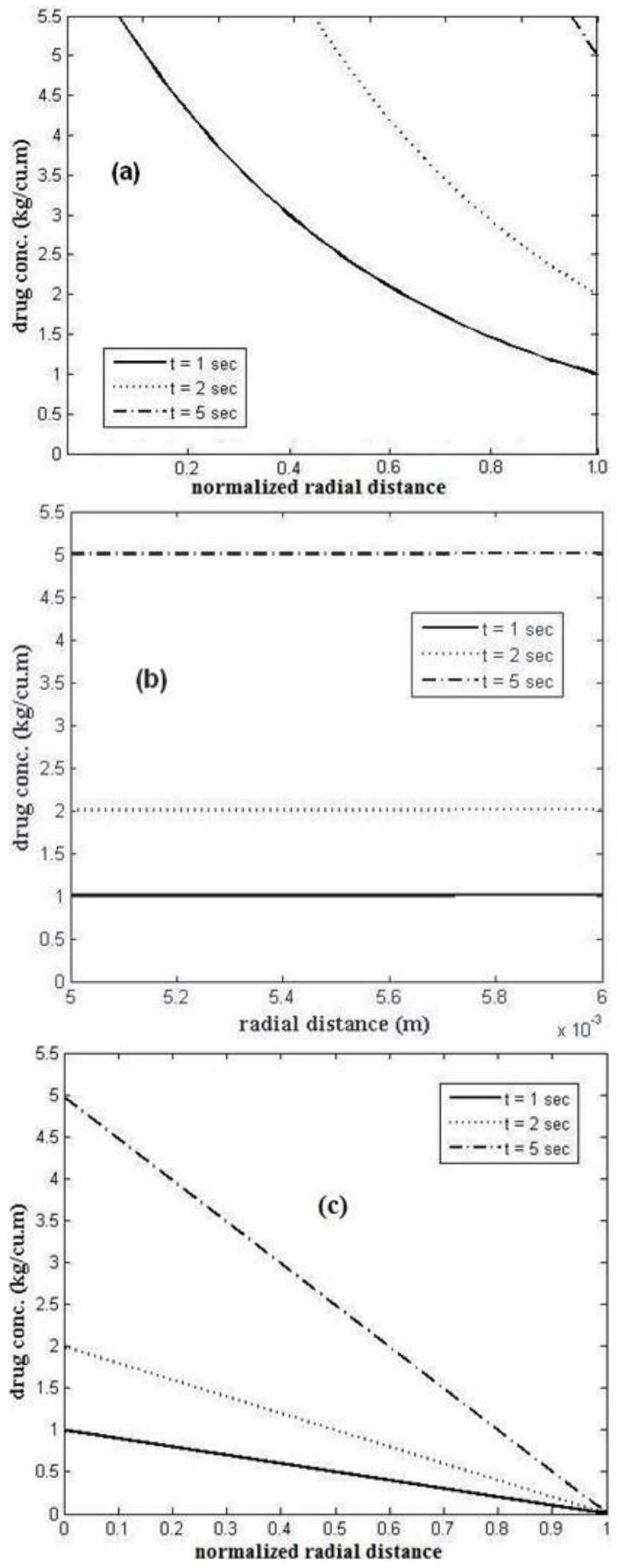

Fig. 3 Drug concentration plots during the negative scan for (a) reservoir, (b) membrane and (c) tissue

effective tool for predicting the drug level for a particular patient and help an oncologist make his decision regarding the optimal parametric values for a particular patient and his condition. The design parameters pertaining to the delivery device such as membrane thickness, drug type and membrane type may be fixed on the basis of the results of drug delivery presented here. The therapeutic concentration - which in this model is a boundary condition - is also a design parameter that needs to be de- 

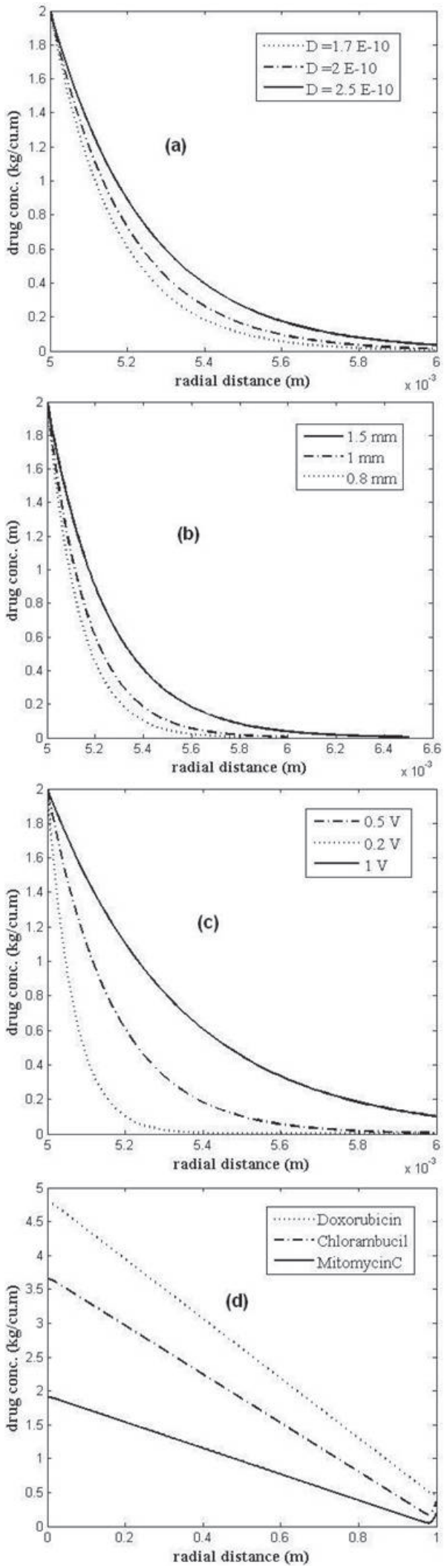

Fig. 4 Parametric studies of (a) polymer diffusivity, (b) membrane thickness, (c) applied voltage and (d) drug type cided upon by the oncologist. Voltage and scan times can be used as regulating parameters. The voltage as shown in our parametric study can be used to enhance the delivery rate. Positive scan time can be increased to increase drug accumulation in the membrane and subsequent higher rate of release. The final set of optimal parameters may be chosen through a detailed understanding of the patient's patho-physiological condition, the behavior of the tumor and after fixing a dosage pattern.

\begin{tabular}{|c|c|c|c|}
\hline \multicolumn{2}{|c|}{ Nomenclature } & \multirow[b]{2}{*}{ concentration variable } & \multirow[b]{2}{*}[\mathrm{kg}/\mathrm{m}^{3}]{} \\
\hline$c$ & $=$ & & \\
\hline$c_{\mathrm{D}}$ & $=$ & concentration of drug & {$\left[\mathrm{kg} / \mathrm{m}^{3}\right]$} \\
\hline$c_{\mathrm{P}}$ & $=$ & concentration of positively charged groups & {$\left[\mathrm{kg} / \mathrm{m}^{3}\right]$} \\
\hline$c_{\mathrm{T}}$ & $=$ & therapeutic concentration & {$\left[\mathrm{kg} / \mathrm{m}^{3}\right]$} \\
\hline$D_{\mathrm{m}}$ & $=$ & diffusivity of drug ions in membrane & {$\left[\mathrm{m}^{2} / \mathrm{s}\right]$} \\
\hline$D_{\mathrm{S}}$ & $=$ & diffusivity of drug ions in solution & {$\left[\mathrm{m}^{2} / \mathrm{s}\right]$} \\
\hline$F$ & $=$ & Faraday's constant & {$[\mathrm{C} / \mathrm{mol}]$} \\
\hline$h$ & $=$ & thickness of membrane & {$[\mathrm{m}]$} \\
\hline$K_{\mathrm{m}}$ & $=$ & Michaelis-Menten constant & {$\left[\mathrm{mol} / \mathrm{m}^{3}\right]$} \\
\hline$k$ & $=$ & Boltzmann constant & $\left.-\mathrm{kg} / \mathrm{s}^{2} / \mathrm{K}\right]$ \\
\hline$k_{\mathrm{f}}$ & $=$ & rate constant of forward reaction & {$\left[\mathrm{s}^{-1}\right]$} \\
\hline$k_{\mathrm{r}}$ & $=$ & rate constant of reverse reaction & {$\left[\mathrm{s}^{-1}\right]$} \\
\hline$n_{0}$ & $=$ & number density of charges species & {$\left[\mathrm{m}^{-3}\right]$} \\
\hline$P_{\mathrm{O}}$ & $=$ & initial conc. of charged groups in membrane & \\
\hline & & & {$\left[\mathrm{kg} / \mathrm{m}^{3}\right]$} \\
\hline$q$ & $=$ & basic charge of an electron & {$[\mathrm{C}]$} \\
\hline$R$ & $=$ & radius of reservoir & [m] \\
\hline$r$ & $=$ & radial variable & [m] \\
\hline$T$ & $=$ & absolute temperature & [K] \\
\hline$t$ & $=$ & time & {$[\mathrm{s}]$} \\
\hline$t_{\mathrm{p}}$ & $=$ & time of positive scan & [s] \\
\hline$t_{\mathrm{n}}$ & $=$ & time of negative scan & [s] \\
\hline$v_{\mathrm{m}}$ & $=$ & volume of membrane & {$\left[\mathrm{m}^{3}\right]$} \\
\hline$v_{\max }$ & $=$ & Michaelis-Menten constant & $\left.\mathrm{ol} /\left(\mathrm{m}^{3} \mathrm{~s}\right)\right]$ \\
\hline$v_{\mathrm{S}}$ & $=$ & volume of solution reservoir & {$\left[\mathrm{m}^{3}\right]$} \\
\hline$x$ & $=$ & spatial variable & {$[\mathrm{m}]$} \\
\hline$z$ & $=$ & valency of ion & [ ] \\
\hline$\varepsilon$ & $=$ & permittivity of medium & [ ] \\
\hline$\varepsilon_{0}$ & $=$ & permittivity of free space & [ ] \\
\hline$\varepsilon_{\mathrm{r}}$ & $=$ & relative permittivity of medium & [ ] \\
\hline$\rho$ & $=$ & charge density of positive charge in membra & ane \\
\hline & & & {$\left[\mathrm{kg} / \mathrm{m}^{3}\right]$} \\
\hline$\psi$ & $=$ & potential distribution function & [V] \\
\hline
\end{tabular}

\section{Literature Cited}

Bolto, B. A. and D. E. Weiss; "Electronic Conduction in Polymers. II. The Electrochemical Reduction of Polypyrrole at Controlled Potential," Aust. J. Chem., 16, 1076-1089 (1963)

Diaz, A. F., K. K. Kanazawa and G. P. Gardini; "Electrochemical Polymerization of Pyrrole," J. Chem. Soc., Chem. Commun., 635-636 (1979)

Frank, A., S. K. Rath and S. S. Venkatraman; "Controlled Release from Bioerodible Polymers: Effect of Drug Type and Polymer Composition," J. Controlled Release, 102, 333-344 (2005)

Gustafson, D. A. and C. A. Pritsos; "Kinetics and Mechanism of Mitomycin C Bioactivation by Xanthene Dehydrogenase under Aerobic and Hypoxic Conditions," Cancer Res., 53, 5470-5474 (1993)

Helfferich, F.; Ion Exchange, McGraw Hill, New York, U.S.A. (1962)

Leong, K. W. and R. Langer; "Polymeric Controlled Drug Delivery," Adv. Drug Delivery Rev., 1, 199-233 (1987)

Lim, J., J. Whitcomb, J. Boyd and J. Varghese; "Transient Finite Element Analysis of Electric Double Layer Using Nernst-PlanckPoisson Equations with a Modified Stern Layer," J. Colloid Interface Sci., 305, 159-174 (2007) 
Ricka, J. and T. Tanaka; "Swelling of Ionic Gels: Quantitative Performance of the Donnan Theory," Macromolecules, 17, 2916-2921 (1984)

Shivanand, P. and O. L. Sprockel; "A Controlled Porosity Drug Delivery System,” Int. J. Pharm., 167, 83-96 (1998)

Skotheim, T. A., R. L. Elsenbaumer and J. R. Reynolds; Handbook of Conducting Polymers, CRC Press, Boca Raton, U.S.A. (1998)
Tanaka, T., D. Fillmaore, S. T. Sun, I. Nishio, G. Swislow and A. Shah; "Phase Transitions in Ionic Gels," Phys. Rev. Lett., 45, 1636-1639 (1980)

Zhang, J., Z. Ye and Y. Lou; "Metabolism of Chlorambucil by Rat-Liver Microsomal Glutathione S-Tranferase," Chem. Biol. Interact., 149, 61-67 (2004) 